\title{
Role of Endoplasmic Reticulum Aminopeptidases in Health and Disease: from Infection to Cancer
}

\author{
Loredana Cifaldi ${ }^{1}$, Paolo Romania ${ }^{1}$, Silvia Lorenzi ${ }^{1}$, Franco Locatelli ${ }^{1,2}$ and Doriana Fruci ${ }^{1, *}$ \\ 1 Paediatric Haematology/Oncology Department, Bambino Gesù Children's Hospital IRCCS, \\ Piazza S. Onofrio 4, Rome 00165, Italy; E-Mails: loredana.cifaldi@opbg.net (L.C.); \\ paolo.romania@opbg.net (P.R.); silvia.lorenzi@opbg.net (S.L.); franco.locatelli@opbg.net (F.L.) \\ University of Pavia, Corso Strada Nuova 65, Pavia I-27100, Italy \\ * Author to whom correspondence should be addressed; E-Mail: doriana.fruci@opbg.net; \\ Tel.: +39-6-68592657; Fax: +39-6-68592904.
}

Received: 1 June 2012; in revised form: 27 June 2012 / Accepted: 29 June 2012 /

Published: 4 July 2012

\begin{abstract}
Endoplasmic reticulum (ER) aminopeptidases ERAP1 and ERAP2 (ERAPs) are essential for the maturation of a wide spectrum of proteins involved in various biological processes. In the ER, these enzymes work in concert to trim peptides for presentation on MHC class I molecules. Loss of ERAPs function substantially alters the repertoire of peptides presented by MHC class I molecules, critically affecting recognition of both NK and $\mathrm{CD}^{+} \mathrm{T}$ cells. In addition, these enzymes are involved in the modulation of inflammatory responses by promoting the shedding of several cytokine receptors, and in the regulation of both blood pressure and angiogenesis. Recent genome-wide association studies have identified common variants of ERAP1 and ERAP2 linked to several human diseases, ranging from viral infections to autoimmunity and cancer. More recently, inhibition of ER peptide trimming has been shown to play a key role in stimulating innate and adaptive anti-tumor immune responses, suggesting that inhibition of ERAPs might be exploited for the establishment of innovative therapeutic approaches against cancer. This review summarizes data currently available for ERAP enzymes in ER peptide trimming and in other immunological and non-immunological functions, paying attention to the emerging role played by these enzymes in human diseases.
\end{abstract}

Keywords: endoplasmic reticulum aminopeptidases; MHC class I; antigen processing; autoimmunity; viral infection; cancer; cancer immunotherapy 


\section{Introduction}

The endoplasmic reticulum (ER) aminopeptidase 1 (ERAP1) and the closely related ER aminopeptidase ERAP2 are zinc-metallopeptidases of the oxytocinase M1 subfamily, which share consensus zinc-binding motifs essential for their enzymatic activity [1]. The human ERAP1 and ERAP2 genes are located on chromosome 5 q15 in the opposite orientation, likely to share regulatory elements. Human ERAP2 has no analogues in rodents (e.g., mouse, rat, rabbit and guinea pig) and evolution studies suggest that it originates from a relatively recent duplication of ERAP1 [2]. These enzymes are normally present in many tissues and are strongly induced after stimulation with type I and type II interferons (IFNs) [3-6] and tumor necrosis factor-alpha (TNF- $\alpha$ ) [7].

ERAP enzymes trim amino acid residues from the $\mathrm{NH}_{2}$ terminus of polypeptides playing an important role in various biological processes (Figure 1). In the ER, ERAP1 and ERAP2 cleave precursors to generate or destroy MHC class I binding peptides [5,8,9]. ERAP1 has also been involved in regulation of innate immune and inflammatory responses by increasing the shedding of cytokine receptors [10-12]. In addition to these immunological functions, ERAP1 and ERAP2 have been implicated in the regulation of angiogenesis and blood pressure $[6,13,14]$. According to these multifunctional properties, ERAP1 is also termed endoplasmic reticulum aminopeptidase associated with antigen processing (ERAAP), adipocyte-derived leucine aminopeptidase (A-LAP), puromycininsensitive leucine-specific aminopeptidase (PILS-AP), or aminopeptidase regulating type I TNF receptor (TNFR1) shedding (ARTS-1), whereas ERAP2 is known as leukocyte-derived arginine aminopeptidase (L-RAP). The terms ERAP1 and ERAP2, approved by the Human Genome Organization Nomenclature Committee, will be used in this review.

\section{Immunological Functions of ER Aminopeptidases}

MHC class I binding peptides are generated by the antigen-processing pathway through a series of sequential steps [15]. In the first step, endogenous proteins are degraded in the cytosol through the proteasome, a multicatalytic complex that generates fragments with hydrophobic or positively charged $\mathrm{COOH}$-terminal anchor residues. Most of these fragments are further processed by cytosolic aminopeptidases, tripeptidyl peptidase II [16-18], leucine aminopeptidase [19,20], bleomycin hydrolase and puromycin-sensitive aminopeptidase [21,22], or directly translocated into the ER lumen by the transporter associated with antigen processing (TAP), an ATP-driven transporter well adapted for the transfer of these precursor peptides [23]. In the ER, the $\mathrm{NH}_{2}$ terminus of these peptides is further trimmed by ER aminopeptidases, i.e., ERAP1 in mice and ERAP1 and ERAP2 in humans, to the proper length for binding to MHC class I molecules and presentation on the cell surface for recognition by NK cells and specific $\mathrm{CD}^{+} \mathrm{T}$ cells (Figure 1a) $[5,8,9]$.

\subsection{Trimming of Antigenic Peptides by ER Aminopeptidases}

ERAP1 efficiently trims peptides of 9-16 amino acids, the length of peptides efficiently transported into the ER by TAP, but spares the longer ones [24]. Of note, its activity is substantially reduced for peptides with proline at position $2\left(\mathrm{X}-\mathrm{P}-\mathrm{X}_{\mathrm{n}}\right)[5,25]$, or for peptides with a size of 8 or 9 amino acids, the optimal length for binding to MHC class I molecules [24]. ERAP1 shows a strong preference for 
peptides with large hydrophobic $\mathrm{COOH}$-terminal residues [24]. ERAP1 activity appears to be also affected by the nature of the internal residues of peptides. In particular, positions 2, 5 and 7 (with position 1 defined as the N-terminal residue of the peptide) were found to be the most important for the peptide sensitivity to ERAP1 degradation [26]. Based on the analogies with TAP and MHC class I preferences, Chang et al. proposed the "molecular ruler" model for ERAP1 [24], that suggests its role in facilitating antigen processing and presentation by trimming precursors transported by TAP to MHC class I binding peptides.

Figure 1. Schematic representation of the multifunctional properties of endoplasmic reticulum (ER) aminopeptidase 1 (ERAP1) and ERAP2. ERAP1 and ERAP2 (ERAPs) are involved in a variety of biological processes including (a) the final trimming of peptides in the endoplasmic reticulum (ER) for presentation on MHC class I molecules; (b) shedding of several cytokine receptors; (c) post-natal angiogenesis; (d) regulation of blood pressure. For details see text.

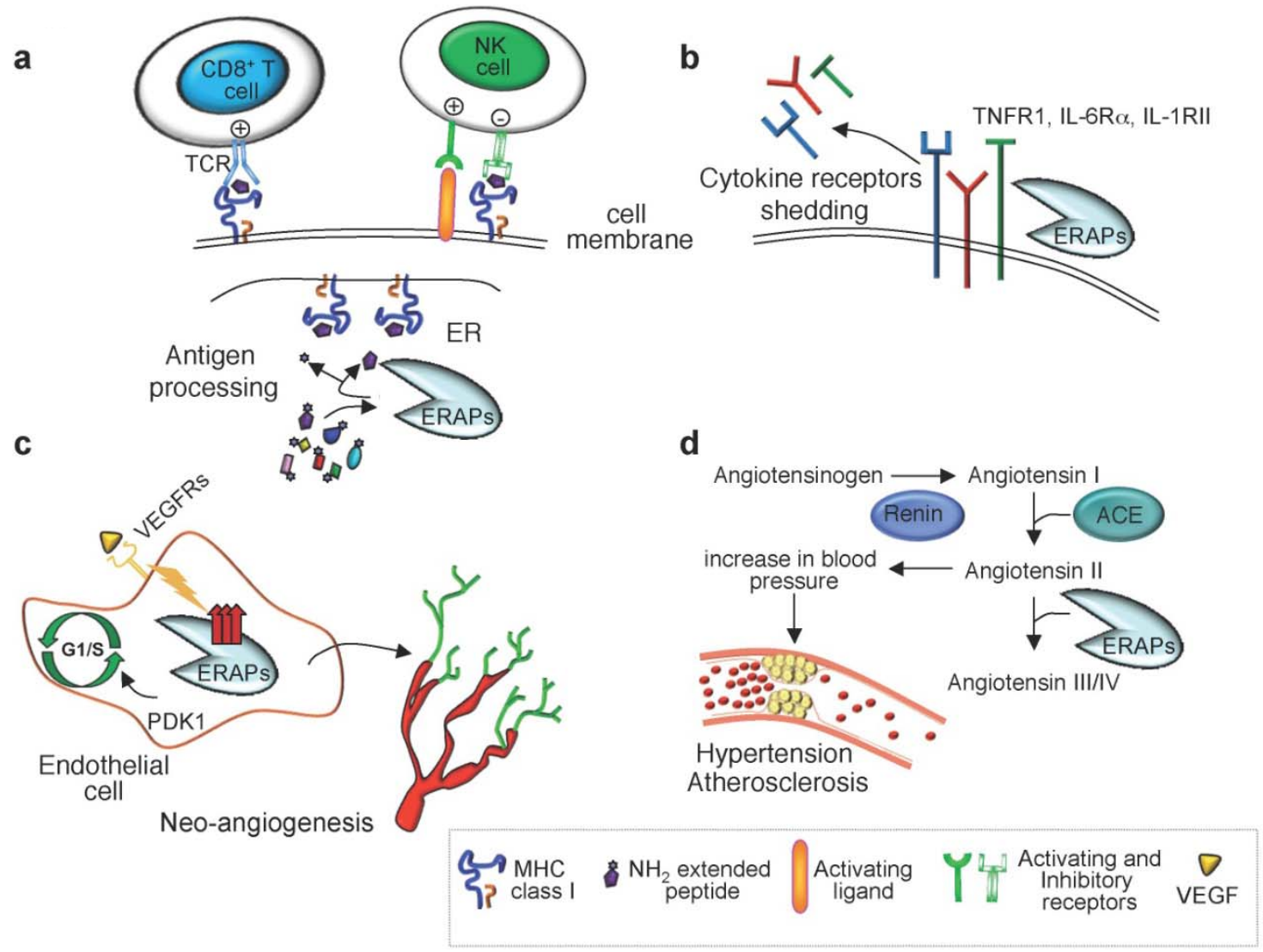

ERAP2 has been shown to cooperate with ERAP1 to trim a large variety of precursor peptides to generate mature epitopes for binding to MHC class I molecules [9]. ERAP2 was found to have distinct specificities for the $\mathrm{N}$-terminal residue of the peptide substrates and to physically associate with ERAP1. This complex is expected to be more efficient than single enzymes in dealing the large number of precursor peptides. To date, there are few studies regarding ERAP2 function.

The substantial contribution of ER peptide trimming to MHC class I antigen processing and presentation has been confirmed in mice lacking ERAP1 generated independently in four 
laboratories [27-30]. Although loss of ERAP1 had a relatively modest effect on the cell surface expression of most MHC class I molecules (a reduction of $20-40 \%$ for $\mathrm{K}^{\mathrm{b}}$ and $\mathrm{D}^{\mathrm{b}}$ class I molecules) [31], immunization of ERAP1 $1^{-/}$mice with wild-type (wt) cells or vice versa, resulted in potent $\mathrm{CD}^{+} \mathrm{T}$ cell responses, suggesting that loss of ERAP1 alters the peptide-MHC (pMHC) class I repertoire not only quantitatively but also qualitatively [27]. Analysis of the individual peptides displayed on the cell surface with a panel of peptide-specific $\mathrm{CD}^{+} \mathrm{T}$ cell hybridomas showed that ERAP1 deficiency left some peptides unaffected, whereas others were either absent or dramatically up-regulated [5,27-32]. Consistent with these findings, mass spectrometry analysis of natural and viral peptides processed in mice lacking ERAP1, revealed that ERAP1 deficiency causes a marked increase in the length of peptides normally presented by MHC class I molecules [33]. Thus, ERAP1 proteolysis determines the characteristic length, as well as the composition of MHC class I binding peptide in the ER.

In addition to classical MHC class I molecules, ERAP1 ${ }^{-/-}$mice also exhibited defects in the surface expression of nonclassical MHC class I molecules Qa-2 and Qa-1 $1^{\mathrm{b}}$, which serve as ligands in both the innate and adaptive immune responses [28]. Yan and collaborators found a significant reduction of the nonclassical class I molecules Qa-2 in ERAP1-deficient splenocytes and dendritic cells as compared with wt cells. Although ERAP1 did not significantly affect the surface expression of Qa-1 molecules, presentation of Qdm, an epitope derived from the signal sequence of classical MHC class I molecules, to Qa-1-restricted CTLs was impaired, suggesting that ERAP1 activity is required for the generation of this epitope. Thus, it is conceivable that reduced ERAP1 function may represent a rate-limiting step in presenting Qdm peptide to Qa- $1^{\mathrm{b}}$-restricted $\mathrm{CD} 8^{+} \mathrm{T}$ cells or $\mathrm{NK}$ cells expressing CD94/NKG2 receptors.

More recently, a new naturally processed Qa- $1^{\mathrm{b}}$ epitope (FL9) derived from the Fam49b protein, has been identified in cells lacking ERAP1 activity by Shastri and colleagues [34]. Unlike the Qa-1 ${ }^{\mathrm{b}}$-Qdm complex, Qa-1 ${ }^{\mathrm{b}}$-FL9 is an immunodominant ligand recognized by $\mathrm{CD}^{+} \mathrm{T}$ cells derived from wt mice immunized with ERAP1 deficient cells. The authors found an abundant fraction of CD $8^{+}$ $\mathrm{T}$ cells specific for the Qa- ${ }^{\mathrm{b}}$-FL9 complex in naive wt mice able to proliferate and efficiently eliminate ERAP1-deficient cells.

\subsection{Cytokine Receptor Shedding}

In addition to antigen processing, ERAP1 has been shown to promote the cleavage of several cytokine cell surface receptors (Figure 1b) [10-12]. Cui et al. demonstrated that ERAP1 binds to the extracellular domain of the TNFR1, facilitating TNFR1 shedding through the formation of a TNFR1/ERAP1 complex. The authors showed that overexpression of ERAP1 produces soluble TNFR1 that competes with cell-surface TNF receptors, thereby attenuating TNF $\alpha$ bioactivity when the levels are elevated, and reconstituting TNF $\alpha$ when the levels have declined [10]. However, several evidences demonstrated that ERAP1 is not required to be catalytically active for this function. Coimmunoprecipitation experiments revealed that ERAP1 can bind to, but not cleave TNFR1. Moreover, ERAP1 does not possess endopeptidase activity and overexpression of ERAP1 catalytic mutants results in an increased TNFR1 shedding. Thus, ERAP1 does not directly catalyze TNFR1 shedding, but may instead promote the activity of a TNFR1 sheddase. 
Subsequently, the same authors showed that ERAP1 modulates the proteolytic cleavage of two other cytokine receptors, the type I IL-6 cytokine receptor (IL-6R $\alpha$ ) [12] and the type II IL-1 decoy receptor (IL-1RII) [11]. Based on these functions, ERAP1 has been proposed to play an important role in regulating innate and inflammatory immune responses by increasing the shedding of these cytokine receptors.

\section{Nonimmunological Functions of ER Aminopeptidases}

ERAP1 and ERAP2 are thought to play a role in the regulation of blood pressure through their involvement in the renin-angiotensin system (Figure 1d). In vitro studies using Chinese Hamster Ovary cells demonstrated that ERAP1 efficiently cleaves angiotensin II to angiotensin III and IV [14], while ERAP2 cleaves angiotensin III to angiotensin IV [6]. In the same system, both enzymes were shown to convert kallidin to bradykinin [6,14].

In addition, ERAP1 has been reported to control post-natal neo-angiogenesis, a physiological process involving the growth of new blood vessels from pre-existing microvessels, by regulating the proliferation and migration of endothelial cells (EC) (Figure 1c) [35-39]. Functional studies revealed that ERAP1 is expressed in ECs during differentiation in vitro and at the site of angiogenesis in vivo upon stimulation with vascular endothelial growth factor (VEGF) [35]. Suppression of ERAP1 expression in ECs inhibited VEGF-stimulated proliferation, migration and vessel network formation in vitro and angiogenesis in vivo [35]. The authors also demonstrated that ERAP1 regulates VEGFstimulated G1/S transition during EC proliferation by binding to phosphatidylinositol-dependent kinase 1 (PDK1). Formation of the ERAP1-PDK1-S6 kinase complex resulted in activation of cyclin-dependent kinase (CDK) $4 / 6$ by phosphorylated S6K that promotes G1/S-phase transition leading to EC proliferation [37]. It was also demonstrated that ERAP1 controls the spreading of ECs by activating endothelial integrins and focal adhesion kinase [36], increasing EC adhesion to the extracellular matrix via RhoA activation [38]. More recently, ERAP1 has also been shown to bind pigpen, a nuclear coiled body component protein involved in angiogenesis [39,40]. However, how pigpen interacts with ERAP1 to promote angiogenesis and whether pigpen is a substrate for ERAP1 remain to be established.

\section{Alteration of ERAP Functions in Human Diseases}

ERAP1 and ERAP2 are highly polymorphic genes. Naturally occurring single nucleotide polymorphisms (SNPs) have been found to correlate with several pathological conditions [41-43]. Because of the role of these enzymes in MHC class I antigen presentation and in all other biological processes, it is conceivable that variations impairing the presentation of pathogen-derived peptides might lead to inadequate immune responses and development of disease. Recent studies that investigated the possible role of ER aminopeptidases in human diseases are summarized below.

\subsection{Hypertension}

By screening for 33 polymorphisms in the human ERAP1 gene, Yamamoto et al. identified the association of ERAP1 variant rs30187 (K528R) with essential hypertension and hypothesized that the 
R528 form of ERAP1 was less active than the K528 form, leading to hypertension due to reduced bradykinin formation and/or lower inactivation of angiotensin II (Figure 1d) [43]. This variant was shown to reduce by $60 \%$ the efficiency of ERAP1 to cleave angiotensin II to angiotensin III and by $70 \%$ to convert kallidin into bradykinin [44]. The ERAP1 rs30187 variant was also found to determine the degree of regression of left ventricular hypertrophy during anti-hypertensive treatment in patients with essential hypertension [45]. The same variant was also associated with haemolytic uremic syndrome (HUS), a disorder characterized by thrombotic microangiopathy frequently caused by gastrointestinal infections with Escherichia coli that produce verotoxins or Shiga toxins [42].

More recently, variants of ERAP1 and ERAP2 have been found to be associated with an increased risk of preeclampsia, a heritable pregnancy specific disorder characterized by new-onset hypertension and proteinuria [46,47]. Of note, ERAP2 expression was previously found altered in first trimester placentas of women prone to develop preeclampsia [48].

\subsection{Bacterial and Viral Infections}

The first evidence demonstrating that ERAP1 can act as a "susceptibility factor" for an infectious organism derived from the observation that ERAP1-deficient mice are not able to process the immunodominant decapeptide HF10 of Toxoplasma gondii and die from overwhelming infection when challenged with this pathogen [49].

ERAP1 plays an important role in immune response to viruses, either enhancing or reducing $\mathrm{CD}^{+}$ T-cell responses to particular viral epitopes. ERAP1-deficient or wt mice infected with lymphocytic choriomeningitis virus (LCMV) showed profound differences in the frequency of $\mathrm{CD}^{+} \mathrm{T}$ cells specific for particular LCMV peptides [30]. In wt mice the magnitude of T-cell responses to different LCMV epitopes followed a hierarchy of immunodominance that is markedly changed in the ERAP1-deficient mice [30].

Draenert et al. reported the first evidence that escape mutations arising in flanking regions of a human immunodeficiency virus (HIV) epitope alter antigen processing mediated by ERAP1 [50]. The authors showed that in HLA-B $57^{+}$-HIV infected individuals, immune selection pressure leads to a mutation from alanine to proline at residue 146 of HIV Gag protein immediately before the $\mathrm{NH}_{2}$ terminus of a dominant HLA-B57-restricted CTL epitope. This mutation was found to prevent the $\mathrm{NH}_{2}$-terminal cleavage by ERAP1, resulting in decreased CTL responses [50]. Of note, it was demonstrated that antigen processing shapes CTL response hierarchies, and that HIV evolution modifies cleavage patterns influencing proteasomal cleavage and, hence, the likelihood of CTL responses toward all epitopes [51]. Interestingly, some variants in ERAP2 have been shown to confer resistance to HIV-1 infection possibly via the presentation of a distinctive peptide repertoire to $\mathrm{CD} 8^{+} \mathrm{T}$ cells [52].

Similarly, in cervical carcinoma induced by persistent infection and malignant transformation of the uterine cervical epithelium by human papillomavirus (HPV), increased cancer metastasis and decreased survival have been reported to be associated with several variants of ERAP1. In this case, down-regulation of ERAP1 may lead to the preferential loading and presentation of non tumor-associated or non HPV-associated peptides, thereby yielding a less immunogenic phenotype and facilitating tumor growth and progression [53]. 
Recently, ERAP1 has been identified as a host target of human cytomegalovirus (HCMV) microRNA miR-US4-1, demonstrating a previously unknown miRNA-based immunoevasion strategy [54]. Viral miR-US4-1 interferes with MHC class I-mediated antigen presentation by targeting ERAP1, thereby influencing the production of many HCMV-derived antigenic peptides during viral infection, which results in immunoevasion of the recognition of viral antigen by $\mathrm{CD}^{+} \mathrm{T}$ cells during the host immune response [54].

\subsection{Autoimmune Diseases}

Recent genome-wide association studies (GWAS) have proven the importance of ERAP1 and ERAP2 genes in conferring susceptibility of individuals to different autoimmune diseases and their linkage with particular MHC class I alleles [41].

The first GWAS realized by the Wellcome Trust Case Control Consortium and the Austro-Anglo-American Spondylitis Consortium has revealed that $26 \%$ of the overall risk to develop ankylosing spondylitis (AS) is accounted for by ERAP1 [55]. The association between AS and ERAP1 variants has been subsequently replicated in other GWAS and several case-control independent studies in Caucasian and Mongolian populations [56-59]. More recently the association with the variant rs30187 has been exclusively found in the cohort of HLA-B27-positive AS patients [60,61]. Of note, the disease-associated ERAP1 variant rs30187 (K528) had faster rate of trimming of peptide precursors than protective ERAP1 variant $[60,62]$. Taken together, these findings support a model in which aberrant peptide trimming by ERAP1 and, as consequence, impaired peptide presentation by HLA-B27 are involved in the AS pathogenesis [60].

Of note, the functional ERAP1 variant (rs30187) linked to AS, hypertension and HUS was associated with type 1 diabetes and multiple sclerosis $[63,64]$. No evidence for an interaction between ERAP1 and MHC class I was observed in diabetic patients [63]. Two other recent GWAS identified ERAP1 as a new psoriasis susceptibility locus and evidence of an interaction between HLA-C*06:02 and ERAP1 was reported [65,66]. Both studied revealed that ERAP1 influences psoriasis susceptibility only in individuals carrying the $H L A-C$ risk allele. A recent meta-analysis of six Crohn's disease GWAS identified ERAP2 as one of the most interesting candidate genes [67].

\subsection{Cancer}

The expression and tissue distribution of ERAP1 and ERAP2 have been evaluated in a large number of tumor cells of lymphoid and non-lymphoid origin compared to their normal counterparts [68-71]. We found that ERAP1 and ERAP2 were expressed in essentially all tumor cell lines examined (melanomas, leukemia-lymphomas and carcinomas of breast, colon, lung, chorion, skin, prostate, cervix, kidney and bladder) at highly variable levels and independently of each other [68]. MHC class I surface expression was significantly correlated with ERAP1, but not with ERAP2, suggesting that ERAP1 has a dominant role in the generation of MHC class I epitopes [68]. In a subsequent study, ERAP1 and ERAP2 were investigated in a large panel of surgically removed normal and neoplastic tissues. In approximately 150 neoplastic lesions, the expression of either or both enzymes was lost, acquired or retained as compared to the normal counterparts, depending on the tumor histotype [69]. Down-regulation of ERAP1 and/or ERAP2 expression was mainly detected in ovary, breast and 
lung carcinomas, whereas an up-regulation of these enzymes was observed in colon and thyroid carcinomas [69]. Of note, ERAP1 and MHC class I were co-ordinately expressed in normal and, to a lesser extent, neoplastic lesions. As expected, the altered expression of ERAPs results in abnormal cell surface expression of MHC class I molecules in tumor cell lines [68]. In the most aggressive type of neuroblastoma cells, ERAP1, ERAP2 as well as MHC class I molecules were expressed at very low levels as consequence of a poor constitutive NF-kB nuclear activity [7].

In a recent study, heterogeneous expression of ERAP1 and ERAP2, ranging from high to very low levels, was detected in 28 melanoma cell lines as compared to primary melanocytes [70]. In most cases, expression of these genes was enhanced by IFN- $\gamma$ treatment, suggesting that it is under control of regulatory mechanisms and that only in rare cases to abnormalities in their sequences [70].

Expression of ERAP1 has been detected in $64 \%$ of endometrial carcinomas and correlated with CA-125 levels, suggesting a role of this enzyme in endometrial cancer cell growth and differentiation [71-73]. The authors also showed that ERAP1 suppresses angiogenesis and endothelial cell migration in human endometrial carcinoma by regulating the angiotensin II concentration [72].

As stated before, variants of ERAP1 have been associated with decreased survival in cervical carcinoma, with ERAP1 loss being an independent predictor for survival, possibly due to the role of ERAP1 as a key determinant of the repertoire of MHC class I-presented peptides $[53,74]$.

All these findings suggest that aberrant expression of ERAP1 may contribute to escape from immune surveillance. To evaluate the relevance of the ER peptide trimming inhibition on tumorigenicity, we stably reduced ERAP1 expression in a murine T-cell lymphoma by ERAP1-targeted small interfering RNA. We demonstrated that interfering with ERAP1 expression ultimately leads to tumor rejection in syngeneic animals by boosting NK cell-, and subsequently T cell-mediated cytolysis (Figure 2) [75]. This rejection was mainly due to NK cell response and depends on the MHC class I peptides presented by ERAP1-silenced tumor cells, because replacement of the endogenous peptides with high-affinity peptides was sufficient to restore an NK protective effect of MHC class I through the NK inhibitory receptor Ly49C/I. In spite of the relatively modest impact on overall MHC class I expression, we demonstrated that ERAP1 inhibition was able to shift the balance between activating and inhibitory signals towards NK cell activation resulting in target cell killing [75]. Thus, this was the first demonstration that tampering with ERAP1 activity via reduction of its expression can result in increased tumor immunogenicity in vivo, and may represent a novel pathway for anti-cancer therapeutic exploitation. 
Figure 2. ERAPs inhibition: a possible novel strategy for anticancer immunotherapy. Antigenic peptides are generated in the cytosol and further trimmed at the $\mathrm{NH}_{2}$-terminus in the endoplasmic reticulum (ER) by ERAP1 and ERAP2 (ERAPs) aminopeptidases before being loaded onto MHC class I molecules. Peptide-MHC (pMHC) class I complexes are presented on the plasma membrane to be recognized by $\mathrm{T}$ cell antigen receptor on $\mathrm{CD}^{+} \mathrm{T}$ cells and by inhibitory receptors on NK cells. In the absence of ERAPs, a distinct repertoire of unstable pMHC class I complexes is produced and exported to the plasma membrane. These unstable complexes are sufficiently conformed to present antigens to $\mathrm{CD} 8^{+} \mathrm{T}$ cells but not enough to inhibit NK cells resulting in tumor-cell killing.

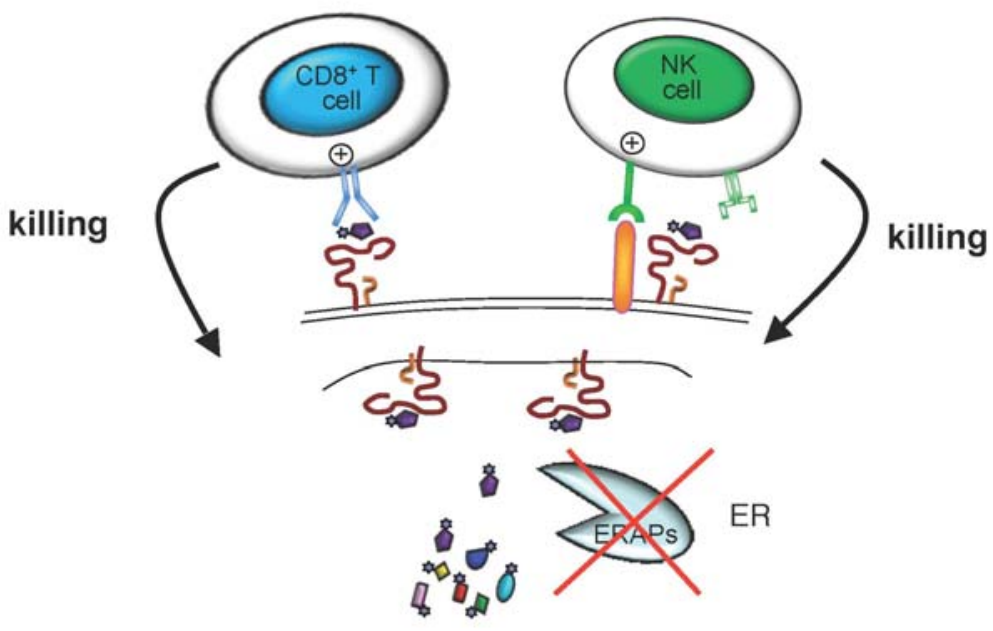

Tumor cells

\section{Conclusions}

In this review, we have briefly summarized the recent knowledge on the biology of ERAP1 and ERAP2 enzymes and their possible links to human diseases.

In autoimmune diseases, association with HLA-class I risk alleles and their interaction with ERAP1 implicates aberrant ER peptide trimming leading to altered peptide presentation as the pathogenic mechanism. Additional functional studies are necessary in order to provide evidence on how ERAPs variants can affect disease predisposition and pathogenesis.

Loss of ERAP1 function has also been shown to substantially affect the presentation of epitopes by classical and nonclassical MHC class I molecules, probably contributing in some cases to the maintenance of chronic infection. In tumor cells, inhibition of ERAP1 expression has been shown to modify tumor immunogenicity by shifting the balance of activating and inhibitory signals towards NK cell activation resulting in target cell killing [75]. In contrast, the same mechanism was not shown in healthy cells lacking NK cell activating ligands such as splenocytes [34]. Based on these findings, it will be of interest to determine whether in humans, as previously shown in mice, manipulation of ERAPs could induce immune-mediated control of cancer. Thus, the possibility of targeting ERAPs through pharmacological or genetic modifications should be considered in order to provide novel anti-tumor immunotherapies. 


\section{Acknowledgments}

This work was supported by the IG grant AIRC (Associazione Italiana per la Ricerca sul Cancro) to D.F. and partly supported by the special project $5 \times 1000$ from AIRC to F.L.

\section{References}

1. Hattori, A.; Matsumoto, H.; Mizutani, S.; Tsujimoto, M. Molecular cloning of adipocyte-derived leucine aminopeptidase highly related to placental leucine aminopeptidase/oxytocinase. J. Biochem. 1999, 125, 931-938.

2. Andres, A.M.; Dennis, M.Y.; Kretzschmar, W.W.; Cannons, J.L.; Lee-Lin, S.Q.; Hurle, B.; Schwartzberg, P.L.; Williamson, S.H.; Bustamante, C.D.; Nielsen, R.; et al. Balancing selection maintains a form of ERAP2 that undergoes nonsense-mediated decay and affects antigen presentation. PLoS Genet. 2010, 6, e1001157. Available online: http://www.plosgenetics.org/ article/info\%3Adoi\%2F10.1371\%2Fjournal.pgen.1001157 (accessed on 29 June 2012).

3. Saric, T.; Chang, S.C.; Hattori, A.; York, I.A.; Markant, S.; Rock, K.L.; Tsujimoto, M.; Goldberg, A.L. An IFN-gamma-induced aminopeptidase in the ER, ERAP1, trims precursors to MHC class I-presented peptides. Nat. Immunol. 2002, 3, 1169-1176.

4. Shin, E.C.; Seifert, U.; Urban, S.; Truong, K.T.; Feinstone, S.M.; Rice, C.M.; Kloetzel, P.M.; Rehermann, B. Proteasome activator and antigen-processing aminopeptidases are regulated by virus-induced type I interferon in the hepatitis C virus-infected liver. J. Interferon Cytokine Res. 2007, 27, 985-990.

5. Serwold, T.; Gonzalez, F.; Kim, J.; Jacob, R.; Shastri, N. ERAAP customizes peptides for MHC class I molecules in the endoplasmic reticulum. Nature 2002, 419, 480-483.

6. Tanioka, T.; Hattori, A.; Masuda, S.; Nomura, Y.; Nakayama, H.; Mizutani, S.; Tsujimoto, M. Human leukocyte-derived arginine aminopeptidase. The third member of the oxytocinase subfamily of aminopeptidases. J. Biol. Chem. 2003, 278, 32275-32283.

7. Forloni, M.; Albini, S.; Limongi, M.Z.; Cifaldi, L.; Boldrini, R.; Nicotra, M.R.; Giannini, G.; Natali, P.G.; Giacomini, P.; Fruci, D. NF-kappaB, and not MYCN, regulates MHC class I and endoplasmic reticulum aminopeptidases in human neuroblastoma cells. Cancer Res. 2010, 70, 916-924.

8. York, I.A.; Chang, S.C.; Saric, T.; Keys, J.A.; Favreau, J.M.; Goldberg, A.L.; Rock, K.L.The ER aminopeptidase ERAP1 enhances or limits antigen presentation by trimming epitopes to 8-9 residues. Nat. Immunol. 2002, 3, 1177-1184.

9. Saveanu, L.; Carroll, O.; Lindo, V.; Del Val, M.; Lopez, D.; Lepelletier, Y.; Greer, F.; Schomburg, L.; Fruci, D.; Niedermann, G.; et al. Concerted peptide trimming by human ERAP1 and ERAP2 aminopeptidase complexes in the endoplasmic reticulum. Nat. Immunol. 2005, 6, 689-697.

10. Cui, X.; Hawari, F.; Alsaaty, S.; Lawrence, M.; Combs, C.A.; Geng, W.; Rouhani, F.N.; Miskinis, D.; Levine, S.J. Identification of ARTS-1 as a novel TNFR1-binding protein that promotes TNFR1 ectodomain shedding. J. Clin. Invest. 2002, 110, 515-526. 
11. Cui, X.; Rouhani, F.N.; Hawari, F.; Levine, S.J. Shedding of the type II IL-1 decoy receptor requires a multifunctional aminopeptidase, aminopeptidase regulator of TNF receptor type 1 shedding. J. Immunol. 2003, 171, 6814-6819.

12. Cui, X.; Rouhani, F.N.; Hawari, F.; Levine, S.J. An aminopeptidase, ARTS-1, is required for interleukin-6 receptor shedding. J. Biol. Chem. 2003, 278, 28677-28685.

13. Sato, Y. Role of aminopeptidase in angiogenesis. Biol. Pharm. Bull. 2004, 27, 772-776.

14. Hattori, A.; Kitatani, K.; Matsumoto, H.; Miyazawa, S.; Rogi, T.; Tsuruoka, N.; Mizutani, S.; Natori, Y.; Tsujimoto, M. Characterization of recombinant human adipocyte-derived leucine aminopeptidase expressed in Chinese hamster ovary cells. J. Biochem. 2000, 128, 755-762.

15. Neefjes, J.; Jongsma, M.L.; Paul, P.; Bakke, O. Towards a systems understanding of MHC class I and MHC class II antigen presentation. Nat. Rev. Immunol. 2011, 11, 823-836.

16. Kloetzel, P.M. Generation of major histocompatibility complex class I antigens: Functional interplay between proteasomes and TPPII. Nat. Immunol. 2004, 5, 661-669.

17. Reits, E.; Neijssen, J.; Herberts, C.; Benckhuijsen, W.; Janssen, L.; Drijfhout, J.W.; Neefjes, J. A major role for TPPII in trimming proteasomal degradation products for MHC class I antigen presentation. Immunity 2004, 20, 495-506.

18. Seifert, U.; Maranon, C.; Shmueli, A.; Desoutter, J.F.; Wesoloski, L.; Janek, K.; Henklein, P.; Diescher, S.; Andrieu, M.; de la Salle, H.; et al. An essential role for tripeptidyl peptidase in the generation of an MHC class I epitope. Nat. Immunol. 2003, 4, 375-379.

19. Beninga, J.; Rock, K.L.; Goldberg, A.L. Interferon-gamma can stimulate post-proteasomal trimming of the $\mathrm{N}$ terminus of an antigenic peptide by inducing leucine aminopeptidase. J. Biol. Chem. 1998, 273, 18734-18742.

20. Paz, P.; Brouwenstijn, N.; Perry, R.; Shastri, N. Discrete proteolytic intermediates in the MHC class I antigen processing pathway and MHC I-dependent peptide trimming in the ER. Immunity 1999, 11, 241-251.

21. Stoltze, L.; Nussbaum, A.K.; Sijts, A.; Emmerich, N.P.; Kloetzel, P.M.; Schild, H. The function of the proteasome system in MHC class I antigen processing. Immunol. Today 2000, 21, 317-319.

22. Stoltze, L.; Schirle, M.; Schwarz, G.; Schroter, C.; Thompson, M.W.; Hersh, L.B.; Kalbacher, H.; Stevanovic, S.; Rammensee, H.G.; Schild, H. Two new proteases in the MHC class I processing pathway. Nat. Immunol. 2000, 1, 413-418.

23. Lauvau, G.; Kakimi, K.; Niedermann, G.; Ostankovitch, M.; Yotnda, P.; Firat, H.; Chisari, F.V.; van Endert, P.M. Human transporters associated with antigen processing (TAPs) select epitope precursor peptides for processing in the endoplasmic reticulum and presentation to T cells. J. Exp. Med. 1999, 190, 1227-1240.

24. Chang, S.C.; Momburg, F.; Bhutani, N.; Goldberg, A.L. The ER aminopeptidase, ERAP1, trims precursors to lengths of MHC class I peptides by a "molecular ruler" mechanism. Proc. Natl. Acad. Sci. USA 2005, 102, 17107-17112.

25. Serwold, T.; Gaw, S.; Shastri, N. ER aminopeptidases generate a unique pool of peptides for MHC class I molecules. Nat. Immunol. 2001, 2, 644-651.

26. Evnouchidou, I.; Momburg, F.; Papakyriakou, A.; Chroni, A.; Leondiadis, L.; Chang, S.C.; Goldberg, A.L.; Stratikos, E. The internal sequence of the peptide-substrate determines its N-terminus trimming by ERAP1. PLoS One 2008, 3, e3658. 
27. Hammer, G.E.; Gonzalez, F.; James, E.; Nolla, H.; Shastri, N. In the absence of aminopeptidase ERAAP, MHC class I molecules present many unstable and highly immunogenic peptides. Nat. Immunol. 2007, 8, 101-108.

28. Yan, J.; Parekh, V.V.; Mendez-Fernandez, Y.; Olivares-Villagomez, D.; Dragovic, S.; Hill, T.; Roopenian, D.C.; Joyce, S.; van Kaer, L. In vivo role of ER-associated peptidase activity in tailoring peptides for presentation by MHC class Ia and class Ib molecules. J. Exp. Med. 2006, 203, 647-659.

29. Firat, E.; Saveanu, L.; Aichele, P.; Staeheli, P.; Huai, J.; Gaedicke, S.; Nil, A.; Besin, G.; Kanzler, B.; van Endert, P.; et al. The role of endoplasmic reticulum-associated aminopeptidase 1 in immunity to infection and in cross-presentation. J. Immunol. 2007, 178, 2241-2248.

30. York, I.A.; Brehm, M.A.; Zendzian, S.; Towne, C.F.; Rock, K.L. Endoplasmic reticulum aminopeptidase 1 (ERAP1) trims MHC class I-presented peptides in vivo and plays an important role in immunodominance. Proc. Natl. Acad. Sci. USA 2006, 103, 9202-9207.

31. Hammer, G.E.; Gonzalez, F.; Champsaur, M.; Cado, D.; Shastri, N. The aminopeptidase ERAAP shapes the peptide repertoire displayed by major histocompatibility complex class I molecules. Nat. Immunol. 2006, 7, 103-112.

32. Blanchard, N.; Shastri, N. Coping with loss of perfection in the MHC class I peptide repertoire. Curr. Opin. Immunol. 2008, 20, 82-88.

33. Blanchard, N.; Kanaseki, T.; Escobar, H.; Delebecque, F.; Nagarajan, N.A.; Reyes-Vargas, E.; Crockett, D.K.; Raulet, D.H.; Delgado, J.C.; Shastri, N. Endoplasmic reticulum aminopeptidase associated with antigen processing defines the composition and structure of MHC class I peptide repertoire in normal and virus-infected cells. J. Immunol. 2010, 184, 3033-3042.

34. Nagarajan, N.A.; Gonzalez, F.; Shastri, N. Nonclassical MHC class Ib-restricted cytotoxic T cells monitor antigen processing in the endoplasmic reticulum. Nat. Immunol. 2012, 13, 579-586.

35. Miyashita, H.; Yamazaki, T.; Akada, T.; Niizeki, O.; Ogawa, M.; Nishikawa, S.; Sato, Y. A mouse orthologue of puromycin-insensitive leucyl-specific aminopeptidase is expressed in endothelial cells and plays an important role in angiogenesis. Blood 2002, 99, 3241-3249.

36. Akada, T.; Yamazaki, T.; Miyashita, H.; Niizeki, O.; Abe, M.; Sato, A.; Satomi, S.; Sato, Y. Puromycin insensitive leucyl-specific aminopeptidase (PILSAP) is involved in the activation of endothelial integrins. J. Cell Physiol. 2002, 193, 253-262.

37. Yamazaki, T.; Akada, T.; Niizeki, O.; Suzuki, T.; Miyashita, H.; Sato, Y. Puromycin-insensitive leucyl-specific aminopeptidase (PILSAP) binds and catalyzes PDK1, allowing VEGF-stimulated activation of S6K for endothelial cell proliferation and angiogenesis. Blood 2004, 104, 2345-52.

38. Suzuki, T.; Abe, M.; Miyashita, H.; Kobayashi, T.; Sato, Y. Puromycin insensitive leucyl-specific aminopeptidase (PILSAP) affects RhoA activation in endothelial cells. J. Cell Physiol. 2007, 211, 708-715.

39. Yoshida, T.; Sato, Y.; Morita, I.; Abe, M. Pigpen, a nuclear coiled body component protein, is involved in angiogenesis. Cancer Sci. 2010, 101, 1170-1176.

40. Abe, M.; Sato, Y. Puromycin insensitive leucyl-specific aminopeptidase (PILSAP) is required for the development of vascular as well as hematopoietic system in embryoid bodies. Genes. Cells 2006, 11, 719-729. 
41. Fierabracci, A.; Milillo, A.; Locatelli, F.; Fruci, D. The putative role of endoplasmic reticulum aminopeptidases in autoimmunity: Insights from genomic-wide association studies. Autoimmun. Rev. 2012, in press.

42. Taranta, A.; Gianviti, A.; Palma, A.; De Luca, V.; Mannucci, L.; Procaccino, M.A.; Ghiggeri, G.M.; Caridi, G.; Fruci, D. Genetic risk factors in typical haemolytic uraemic syndrome. Nephrol. Dial. Transplant. 2009, 24, 1851-1857.

43. Yamamoto, N.; Nakayama, J.; Yamakawa-Kobayashi, K.; Hamaguchi, H.; Miyazaki, R.; Arinami, T. Identification of 33 polymorphisms in the adipocyte-derived leucine aminopeptidase (ALAP) gene and possible association with hypertension. Hum. Mutat. 2002, 19, 251-257.

44. Goto, Y.; Hattori, A.; Ishii, Y.; Mizutani, S.; Tsujimoto, M. Enzymatic properties of human aminopeptidase A. Regulation of its enzymatic activity by calcium and angiotensin IV. J. Biol. Chem. 2006, 281, 23503-23513.

45. Hallberg, P.; Lind, L.; Michaelsson, K.; Kurland, L.; Kahan, T.; Malmqvist, K.; Ohman, K.P.; Nystrom, F.; Liljedahl, U.; Syvanen, A.C.; et al. Adipocyte-derived leucine aminopeptidase genotype and response to antihypertensive therapy. BMC Cardiovasc. Disord. 2003, 3, 11.

46. Hill, L.D.; Hilliard, D.D.; York, T.P.; Srinivas, S.; Kusanovic, J.P.; Gomez, R.; Elovitz, M.A.; Romero, R.; Strauss, J.F. Fetal ERAP2 variation is associated with preeclampsia in African Americans in a case-control study. BMC Med. Genet. 2011, 12, 64.

47. Johnson, M.P.; Roten, L.T.; Dyer, T.D.; East, C.E.; Forsmo, S.; Blangero, J.; Brennecke, S.P.; Austgulen, R.; Moses, E.K. The ERAP2 gene is associated with preeclampsia in Australian and Norwegian populations. Hum. Genet. 2009, 126, 655-666.

48. Founds, S.A.; Conley, Y.P.; Lyons-Weiler, J.F.; Jeyabalan, A.; Hogge, W.A.; Conrad, K.P. Altered global gene expression in first trimester placentas of women destined to develop preeclampsia. Placenta 2009, 30, 15-24.

49. Blanchard, N.; Gonzalez, F.; Schaeffer, M.; Joncker, N.T.; Cheng, T.; Shastri, A.J.; Robey, E.A.; Shastri, N. Immunodominant, protective response to the parasite Toxoplasma gondii requires antigen processing in the endoplasmic reticulum. Nat. Immunol. 2008, 9, 937-944.

50. Draenert, R.; Le Gall, S.; Pfafferott, K.J.; Leslie, A.J.; Chetty, P.; Brander, C.; Holmes, E.C.; Chang, S.C.; Feeney, M.E.; Addo, M.M.; et al. Immune selection for altered antigen processing leads to cytotoxic T lymphocyte escape in chronic HIV-1 infection. J. Exp. Med. 2004, 199, 905-915.

51. Tenzer, S.; Wee, E.; Burgevin, A.; Stewart-Jones, G.; Friis, L.; Lamberth, K.; Chang, C.H.; Harndahl, M.; Weimershaus, M.; Gerstoft, J. Antigen processing influences HIV-specific cytotoxic T lymphocyte immunodominance. Nat. Immunol. 2009, 10, 636-646.

52. Cagliani, R.; Riva, S.; Biasin, M.; Fumagalli, M.; Pozzoli, U.; Lo Caputo, S.; Mazzotta, F.; Piacentini, L.; Bresolin, N.; Clerici, M.; et al. Genetic diversity at endoplasmic reticulum aminopeptidases is maintained by balancing selection and is associated with natural resistance to HIV-1 infection. Hum. Mol. Genet. 2010, 19, 4705-4714.

53. Mehta, A.M.; Jordanova, E.S.; Corver, W.E.; van Wezel, T.; Uh, H.W.; Kenter, G.G.; Fleuren, G.J. Single nucleotide polymorphisms in antigen processing machinery component ERAP1 significantly associate with clinical outcome in cervical carcinoma. Genes. Chromosomes Cancer 2009, 48, 410-418. 
54. Kim, S.; Lee, S.; Shin, J.; Kim, Y.; Evnouchidou, I.; Kim, D.; Kim, Y.K.; Kim, Y.E.; Ahn, J.H.; Riddell, S.R.; et al. Human cytomegalovirus microRNA miR-US4-1 inhibits $\mathrm{CD} 8^{(+)} \mathrm{T}$ cell responses by targeting the aminopeptidase ERAP1. Nat. Immunol. 2011, 12, 984-991.

55. Burton, P.R.; Clayton, D.G.; Cardon, L.R.; Craddock, N.; Deloukas, P.; Duncanson, A.; Kwiatkowski, D.P.; McCarthy, M.I.; Ouwehand, W.H.; Samani, N.J.; et al. Association scan of 14,500 nonsynonymous SNPs in four diseases identifies autoimmunity variants. Nat. Genet. 2007, 39, 1329-1337.

56. Maksymowych, W.P.; Inman, R.D.; Gladman, D.D.; Reeve, J.P.; Pope, A.; Rahman, P. Association of a specific ERAP1/ARTS1 haplotype with disease susceptibility in ankylosing spondylitis. Arthritis Rheum. 2009, 60, 1317-1323.

57. Davidson, S.I.; Wu, X.; Liu, Y.; Wei, M.; Danoy, P.A.; Thomas, G.; Cai, Q.; Sun, L.; Duncan, E.; Wang, N. Association of ERAP1, but not IL23R, with ankylosing spondylitis in a Han Chinese population. Arthritis Rheum. 2009, 60, 3263-3268.

58. Choi, C.B.; Kim, T.H.; Jun, J.B.; Lee, H.S.; Shim, S.C.; Lee, B.; Pope, A.; Uddin, M.; Rahman, P.; Inman, R.D. ARTS1 polymorphisms are associated with ankylosing spondylitis in Koreans. Ann. Rheum. Dis. 2010, 69, 582-584.

59. Harvey, D.; Pointon, J.J.; Evans, D.M.; Karaderi, T.; Farrar, C.; Appleton, L.H.; Sturrock, R.D.; Stone, M.A.; Oppermann, U.; Brown, M.A.; Wordsworth, B.P.; et al. Investigating the genetic association between ERAP1 and ankylosing spondylitis. Hum. Mol. Genet. 2009, 18, 4204-4212.

60. Evans, D.M.; Spencer, C.C.; Pointon, J.J.; Su, Z.; Harvey, D.; Kochan, G.; Oppermann, U.; Dilthey, A.; Pirinen, M.; Stone, M.A. Interaction between ERAP1 and HLA-B27 in ankylosing spondylitis implicates peptide handling in the mechanism for HLA-B27 in disease susceptibility. Nat. Genet. 2011, 43, 761-767.

61. Pazar, B.; Safrany, E.; Gergely, P.; Szanto, S.; Szekanecz, Z.; Poor, G. Association of ARTS1 gene polymorphisms with ankylosing spondylitis in the Hungarian population: the rs27044 variant is associated with HLA-B*2705 subtype in Hungarian patients with ankylosing spondylitis. J. Rheumatol. 2010, 37, 379-384.

62. Evnouchidou, I.; Kamal, R.P.; Seregin, S.S.; Goto, Y.; Tsujimoto, M.; Hattori, A.; Voulgari, P.V.; Drosos, A.A.; Amalfitano, A.; York, I.A.; et al. Cutting Edge: Coding single nucleotide polymorphisms of endoplasmic reticulum aminopeptidase 1 can affect antigenic peptide generation in vitro by influencing basic enzymatic properties of the enzyme. J. Immunol. 2011, 186, 1909-1913.

63. Fung, E.Y.; Smyth, D.J.; Howson, J.M.; Cooper, J.D.; Walker, N.M.; Stevens, H.; Wicker, L.S.; Todd, J.A. Analysis of 17 autoimmune disease-associated variants in type 1 diabetes identifies 6q23/TNFAIP3 as a susceptibility locus. Genes. Immun. 2009, 10, 188-191.

64. Guerini, F.R.; Cagliani, R.; Forni, D.; Agliardi, C.; Caputo, D.; Cassinotti, A.; Galimberti, D.; Fenoglio, C.; Biasin, M.; Asselta, R.; et al. A functional variant in ERAP1 predisposes to multiple sclerosis. PLoS One 2012, 7, e29931.

65. Strange, A.; Capon, F.; Spencer, C.C.; Knight, J.; Weale, M.E.; Allen, M.H.; Barton, A.; Band, G.; Bellenguez, C.; Bergboer, J.G.; et al. A genome-wide association study identifies new psoriasis susceptibility loci and an interaction between HLA-C and ERAP1. Nat. Genet. 2010, 42, 985-990. 
66. Sun, L.D.; Cheng, H.; Wang, Z.X.; Zhang, A.P.; Wang, P.G.; Xu, J.H.; Zhu, Q.X.; Zhou, H.S.; Ellinghaus, E.; Zhang, F.R.; et al. Association analyses identify six new psoriasis susceptibility loci in the Chinese population. Nat. Genet. 2010, 42, 1005-1009.

67. Franke, A.; McGovern, D.P.; Barrett, J.C.; Wang, K.; Radford-Smith, G.L.; Ahmad, T.; Lees, C.W.; Balschun, T.; Lee, J.; Roberts, R.; et al. Genome-wide meta-analysis increases to 71 the number of confirmed Crohn's disease susceptibility loci. Nat. Genet. 2010, 42, 1118-1125.

68. Fruci, D.; Ferracuti, S.; Limongi, M.Z.; Cunsolo, V.; Giorda, E.; Fraioli, R.; Sibilio, L.; Carroll, O.; Hattori, A.; van Endert, P.M.; et al. Expression of endoplasmic reticulum aminopeptidases in EBV-B cell lines from healthy donors and in leukemia/lymphoma, carcinoma, and melanoma cell lines. J. Immunol. 2006, 176, 4869-4879.

69. Fruci, D.; Giacomini, P.; Nicotra, M.R.; Forloni, M.; Fraioli, R.; Saveanu, L.; van Endert, P.; Natali, P.G. Altered expression of endoplasmic reticulum aminopeptidases ERAP1 and ERAP2 in transformed non-lymphoid human tissues. J. Cell Physiol. 2008, 216, 742-749.

70. Kamphausen, E.; Kellert, C.; Abbas, T.; Akkad, N.; Tenzer, S.; Pawelec, G.; Schild, H.; van Endert, P.; Seliger, B. Distinct molecular mechanisms leading to deficient expression of ER-resident aminopeptidases in melanoma. Cancer Immunol. Immunother. 2010, 59, 1273-1284.

71. Kazeto, H.; Nomura, S.; Ito, N.; Ito, T.; Watanabe, Y.; Kajiyama, H.; Shibata, K.; Ino, K.; Tamakoshi, K.; Hattori, A.; et al. Expression of adipocyte-derived leucine aminopeptidase in endometrial cancer. Association with tumor grade and CA-125. Tumour. Biol. 2003, 24, 203-208.

72. Watanabe, Y.; Shibata, K.; Kikkawa, F.; Kajiyama, H.; Ino, K.; Hattori, A.; Tsujimoto, M.; Mizutani, S. Adipocyte-derived leucine aminopeptidase suppresses angiogenesis in human endometrial carcinoma via renin-angiotensin system. Clin. Cancer Res. 2003, 9, 6497-6503.

73. Shibata, K.; Kikkawa, F.; Mizokami, Y.; Kajiyama, H.; Ino, K.; Nomura, S.; Mizutani, S. Possible involvement of adipocyte-derived leucine aminopeptidase via angiotensin II in endometrial carcinoma. Tumour. Biol. 2005, 26, 9-16.

74. Mehta, A.M.; Jordanova, E.S.; Kenter, G.G.; Ferrone, S.; Fleuren, G.J. Association of antigen processing machinery and HLA class I defects with clinicopathological outcome in cervical carcinoma. Cancer Immunol. Immunother. 2008, 57, 197-206.

75. Cifaldi, L.; Lo Monaco, E.; Forloni, M.; Giorda, E.; Lorenzi, S.; Petrini, S.; Tremante, E.; Pende, D.; Locatelli, F.; Giacomini, P.; et al. Natural killer cells efficiently reject lymphoma silenced for the endoplasmic reticulum aminopeptidase associated with antigen processing. Cancer Res. 2011, 71, 1597-1606.

(C) 2012 by the authors; licensee MDPI, Basel, Switzerland. This article is an open access article distributed under the terms and conditions of the Creative Commons Attribution license (http://creativecommons.org/licenses/by/3.0/). 